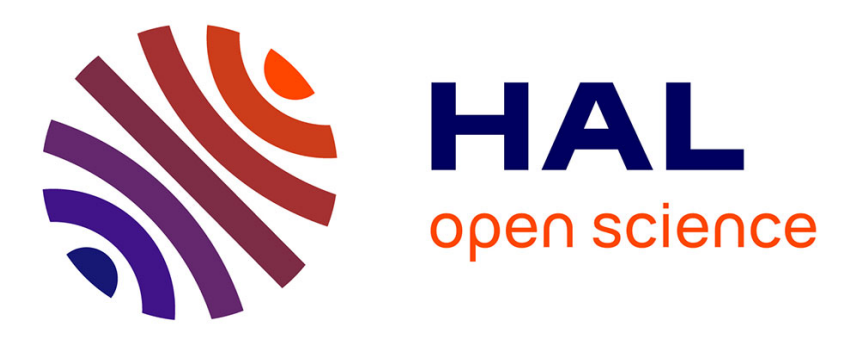

\title{
Numerical modelling of heat transfers between inductively heated metallic and dielectric phases
}

Remi Bourrou, Olga Budenkova, A. Gagnoud, Christophe Lafon

\section{To cite this version:}

Remi Bourrou, Olga Budenkova, A. Gagnoud, Christophe Lafon. Numerical modelling of heat transfers between inductively heated metallic and dielectric phases. COMPEL: The International Journal for Computation and Mathematics in Electrical and Electronic Engineering, 2020, 39 (1), pp.108-116. 10.1108/COMPEL-05-2019-0218 . hal-03017050

\section{HAL Id: hal-03017050 https://hal.science/hal-03017050}

Submitted on 20 Nov 2020

HAL is a multi-disciplinary open access archive for the deposit and dissemination of scientific research documents, whether they are published or not. The documents may come from teaching and research institutions in France or abroad, or from public or private research centers.
L'archive ouverte pluridisciplinaire HAL, est destinée au dépôt et à la diffusion de documents scientifiques de niveau recherche, publiés ou non, émanant des établissements d'enseignement et de recherche français ou étrangers, des laboratoires publics ou privés. 
Before revision, as submitted to COMPEL-The international journal for computation and mathematics in electrical and electronic engineering, year 2020. Be aware of changes due to revision process

Printed: Bourrou, R., Budenkova, O., Lafon, C. and Gagnoud, A. (2020), "Numerical modelling of heat transfers between inductively heated metallic and dielectric phases", COMPEL - The international journal for computation and mathematics in electrical and electronic engineering, Vol. 39 No. 1, pp. 108-116. https://doi.org/10.1108/COMPEL-05-2019-0218

\title{
Numerical Modeling of Heat Transfers Between Inductively Heated Metallic and Dielectric Phases
}

Rémi Bourrou

CEA Marcoule, Bagnols-sur-Ceze, France and SIMAP, AQ: 1 SaintMartin d'Heres, France

Olga Budenkova

SIMAP, Saint Martin d'Heres, France

Christophe Lafon

CEA Marcoule, Bagnols-sur-Ceze, France, and

Annie Gagnoud

SIMAP, Saint Martin d'Heres, France

\begin{abstract}
Heating with a low-frequency induction is a key phenomenon in a process dedicated to the treatment of nuclear wastes. This papers presents a step of the numerical model being developed in order to study this process. A hydrodynamic model for the processing of a liquid charge consisting of a metallic phase and a dielectric one is developed based on a Volume Of Fluid approach coupled with electromagnetic calculations. The latter allows one to calculate the distribution of the Joule heating in the setup and radiative heat exchange inside the crucible is accounted with a Surface-To-Surface (S2S) model coupled with VOF. Numerical results are compared with the measures obtained on the prototype of the process. The results are in good agreement but the model needs to be improved in order to consider the varying viscosity of the glass. The usage of a radiation model coupled to the VOF model is not common for studies of materials melted by electromagnetic induction. This paper demonstrate the feasibility of this approach.
\end{abstract}

\section{INTRODUCTION}

In the context of nuclear waste treatment, Andra, the CEA and the Orano company are currently developing a process where metallic and dielectric radioactive wastes are treated simultaneously in order to produce a package compatible with long term storage in a geological repository. In this process, a heavier metal phase, which is heated and stirred electromagnetically, is covered with a conditioning glass where the dielectric wastes are incorporated in the form of ashes.

For proper distribution of the radionuclides in the package, it is crucial to maintain a good stirring of both materials and therefore ensure that the temperature of the glass remains high enough in order to have there a low viscosity which varies strongly with temperature. The prototype of the set-up (Figure 1), is currently charged with a maximal admissible value of glass.

A numerical simulation is carried out in order to understand the phenomena taking place in the process. One of the crucial points of the numerical modelling is the representation of the deformation of the metal phase by the electromagnetic pressure. That leads to the formation of a steep dome that can emerge from the glass phase. The modelling of this phenomenon is performed using a three-fluid Volume Of Fluid model (VOF) in a 2D axisymmetric configuration, and is detailed in [1].

In order to later replicate the variation of viscosity of the glass, the calculation of heat transfers is implemented in this MHD model. Here the implementation of the numerical treatment of the radiative heat transfer between the free surface of the liquids and the set-up 
walls above it is presented along with a strategy for the calculation of the heat transfers. The results of the model are compared to measurements done on the prototype of the process.

\section{NUMERICAL MODELING}

Apart from the magnetic pressure that leads to the deformation of the shape for the liquid metal volume and the stirring of the metallic phase, the electric resistance and the eddy current leads to the heating of the metallic phase. Further, the heat is transported to the glass and the upper part of the crucible via convection and radiation and also to the external cooling system.

\section{Convective heat transport above the liquids}

The total height of the prototype set-up is almost 3 times larger than that of the crucible and air fills the volume above the liquids. It is not remained still, the air motion is caused by its interaction with the moving liquids and also by a large temperature gradient between the melt and the cooled walls (around $1000 \mathrm{~K}$ ) that leads to a strong natural convection. In order to resolve the interaction of the forced and buoyancy air motion, a lot of mesh cells along with a small time stepping would be required in the numerical model that would increase the calculation time enormously.

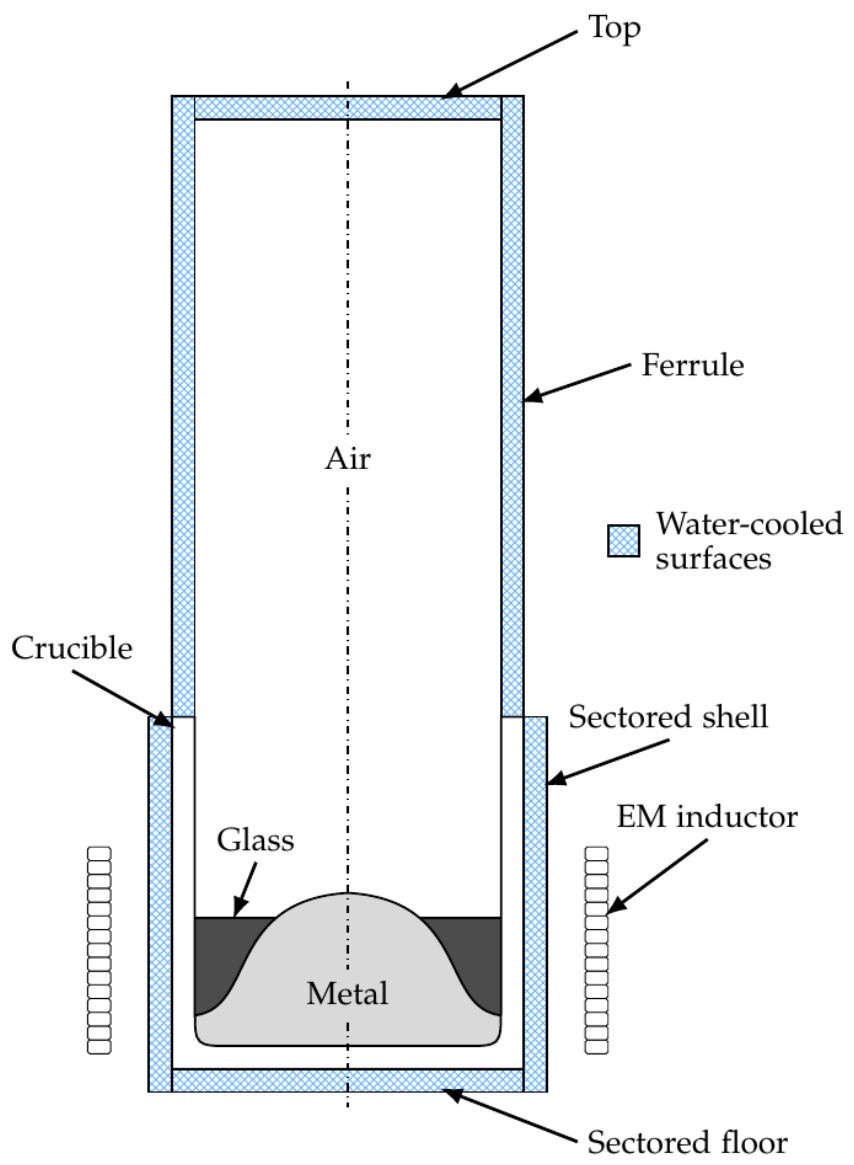

Figure 1. Sketch of the prototype of the process
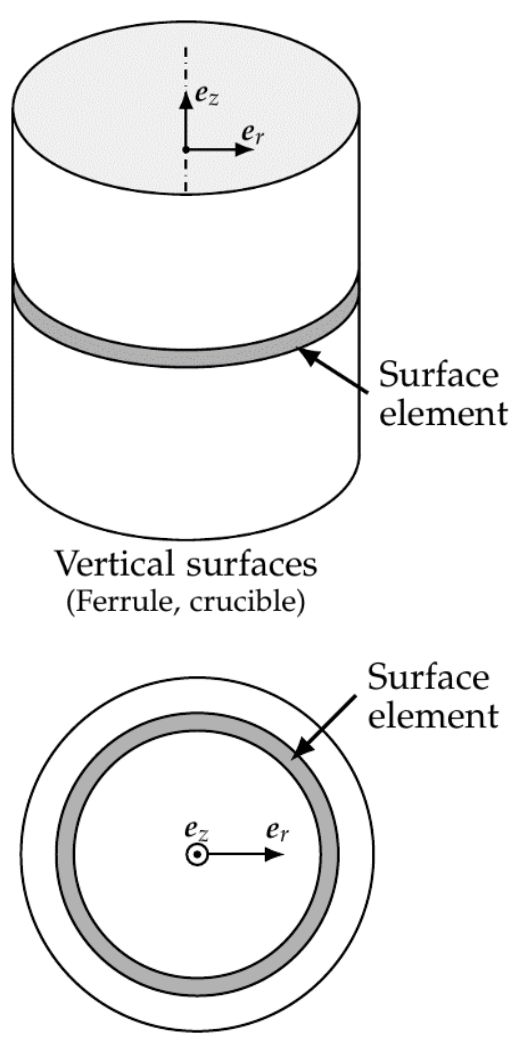

Horizontal surfaces

(Top, liquids free surface)

Figure 2. Surface discretization for the S2S model 
Yet, estimation shows that the convective heat exchange between the free surface of the liquids and the internal surface of the set-up is only $8 \%$ of the radiative one. Since the flow and temperature of the air is not of interest, the convective heat transfer is neglected in the present model, in order to keep a reasonable calculation time.

Radiative heat transport above the liquids

The main problem when using the VOF model with heat transfers in this case, is the thermal radiation of the liquids. Indeed, since the free surface of the liquids is not treated as a surface but is presented as a transition layer between two phases, standard boundary conditions or Surface To Surface (S2S) models cannot be used.

Although other radiation models compatible with the VOF model exists, they are aimed to represent radiation in semi-transparent materials and hardly can be used for the case of opaque materials for several reasons:

- For opaque materials the absorption coefficients, as a limit, should be equal to infinity, i.e. a very larger value should be set for it in the model.

- These models necessitate a large number of iterations in order to converge when using very large absorption coefficients.

- If the absorption coefficient is not set to infinity, these models produces radiation inside the materials, artificially increasing the thermal conductivity.

The solution chosen to overcome these problems is to apply the principle of the $\mathrm{S} 2 \mathrm{~S}$ radiation to the VOF model. To do so, we consider that, in the liquids, the radiative heat transport exists only at the free surface (the air bubbles trapped in the liquids are supposed isothermal) and that the surface radiation flux can be assimilated to a volumetric source applied to the first mesh cell of the free surface.

This hypothesis has been tested on a simple 1D case using finite volume formulation and the VOF model, and compared to theoretical results. This shows that the hypothesis is valid as long as the size of the mesh cells is fine enough (around $1 \times 1 \mathrm{~mm}$ here, due to the requirement of the VOF model) and if the mesh cell containing the interface have a volumetric fraction of the radiating material above 0.2 . If it does not, the second cell deeper in the material should be used to obtain a result closer to the theory.

In order to calculate the radiation fluxes between the crucible wall, the cooling elements, and the free surface of the liquids, all of which are assumed to be grey and diffuse, the view factors between these elements must be calculated. For this, each surface is discretised in elementary faces which are considered isothermal. To simplify the calculation of the view factors, the free surface of the liquids is considered to be flat, and the other elements being perfect cylinders and disk (Figure 2), respectively that allow using of analytical formulas.

Then a system of equations can be written for radiosities $J_{i}$ for each elementary face, which represent the radiative energy leaving it:

$$
J_{i}=\epsilon_{i} \sigma T_{i}^{4}+\left(1-\epsilon_{i}\right) \sum_{j=0}^{N} J_{j} F_{i \rightarrow j}
$$

$F_{i \rightarrow j}$ being the view factor from the elementary face $i$ to $j, \epsilon_{i}$ its emissivity and $T_{i}$ its temperature which should be found as well. Radiosities and the resulting radiative flux $\varphi_{R, i}$ at the elementary face $i$ are related as follows:

$$
\varphi_{R, i}=\sum_{j=0}^{N} J_{j} F_{i \rightarrow j}-J_{i}
$$

Another equation for the temperature of each elementary face can be written using the heat 
balance between the net radiative flux at the surface and the flux removed from it by the external water-cooling. The temperature of the water $T_{\text {cool }}$ is known from the measurement made on the prototype and the convective heat transfer coefficient $h_{\text {cool }}$ is estimated using appropriate relation for the known regime of the external flow. Consequently, another equation for each elementary surface is:

$$
T_{i}=T_{\text {cool }}+\varphi_{R, i} / h_{\text {cool }}
$$

The system of equation (1)-(3) is non-linear and solved iteratively outside of Fluent. To get the temperature of the free surface of the liquids and the material distribution along the surface (thus, the distribution of emissivity), which are the inputs of this system, the free surface of the liquids must be detected. In this case, since the mesh is only composed of squares aligned with the crucible, the detection consists in the extraction of the cell containing an interface with the highest $z$ coordinate, for every column of mesh cell. As a result of the equation system, the temperatures of the ferrule and the top surface are found and the resulting radiative heat flux is calculated for the free surface of the liquids and the crucible.

\section{Modeling strategy}

In order to reduce the calculation time, the heat transfers are not calculated along with the fluid flow since the characteristic time of the two phenomena are very different: the order of magnitude for the fluid flow is a few seconds, but several hours for the heat transfers is needed because of a large quantity of materials involved. First, only the shape of the surface of the metal, and the flow in the fluids are calculated considering a constant temperature [1].

After that, the velocity of the fluids is saved apart and set to 0 for the next stage of calculation in order to calculate the conductive heat transfers in the fluids along with the radiative transfers through the free surface with accounting for the shape of the materials. This is performed in order to quickly obtain an initial guess on the temperature using a steady state calculation.

Then, the velocity of the materials is restored from the saved one and the calculation continues considering also the convective transfers in the liquids, using an unsteady formulation. At the end of this step, the results represent the temperature and fluxes distribution in the process, if we consider that the properties of the materials do not vary with the temperature.

On the numerical model, the sum of the cooling fluxes slightly differs from the injected power in the load. This phenomenon occurs only with the fluid flow activated and comes from the advection of the temperature near the interfaces between the fluids, due to the huge anisotropy of the coefficients in these area when using the VOF model, especially between the liquids and the air. This imbalance depending mainly on the position of the liquids, only results with flux imbalance below $1 \%$ were accepted.

\section{RESULTS}

First, we can notice that the electromagnetic model outputs a different total Joule power in the load than what is measured with the cooling fluxes on the prototype. This probably comes from errors on the measures of the electrical parameters of the generator - taken as an input for the numerical model - , or in the measurement of the cooling fluxes. Therefore, the comparison is made on the flux repartition rather than the absolute values. Table 1 shows that the flux repartition is close to what is observed on the prototype.

Table 1: Comparison of the repartition of the cooling fluxes between the numerical model and the prototype

\begin{tabular}{l|l|l|l|l} 
Flux & Top & Ferrule & Side & Floor
\end{tabular}




\begin{tabular}{r|c|c|c|c|}
\hline \multirow{2}{*}{ Prototype } & $3.7 \mathrm{~W}$ & $26.98 \mathrm{~kW}$ & $53.42 \mathrm{~kW}$ & $8.42 \mathrm{~kW}$ \\
\cline { 2 - 5 } & $4.0 \%$ & $29.2 \%$ & $57.7 \%$ & $9.1 \%$ \\
\hline \multirow{2}{*}{ Numerical model } & $3.07 \mathrm{~kW}$ & $24.58 \mathrm{~kW}$ & $58.91 \mathrm{~kW}$ & $7.42 \mathrm{~kW}$ \\
\cline { 2 - 5 } & $3.7 \%$ & $29.5 \%$ & $58.9 \%$ & $8.9 \%$ \\
\hline
\end{tabular}

The temperature in the liquids is essentially homogenous (Figure 3), at the exception of the free surface of the glass, near the crucible. This is due to the velocity being very low on this part of the glass, and the upper part of the crucible being colder. This behaviour is also observed on the prototype of the process, and the solidification of the free surface starts from this point when it occurs. A thermocouple inside the crucible gives a temperature of approximately $1252{ }^{\circ} \mathrm{C}$ on the prototype, and the same point is at $1243{ }^{\circ} \mathrm{C}$ in the results of the numerical model which is very close.

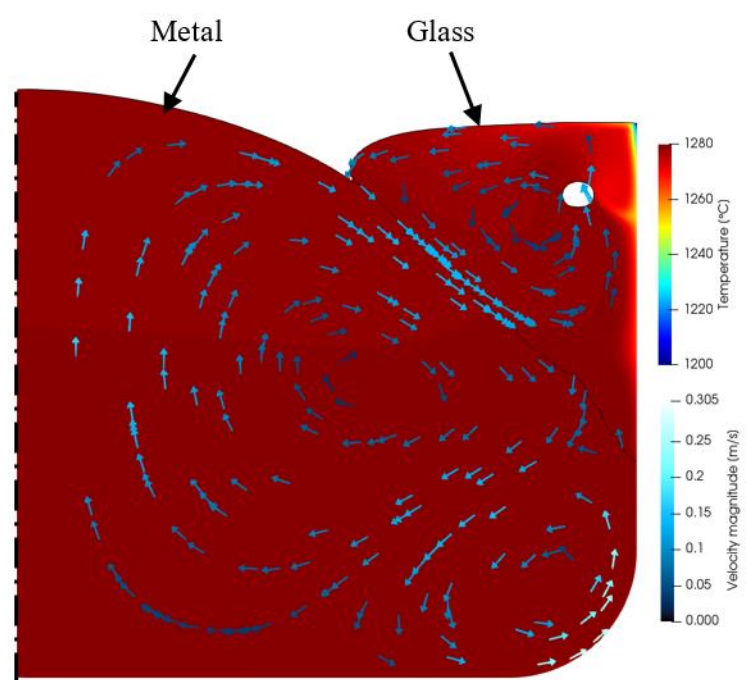

Figure 3. Temperature distribution and velocity vectors in the liquids for $25 \mathrm{~kg}$ of glass

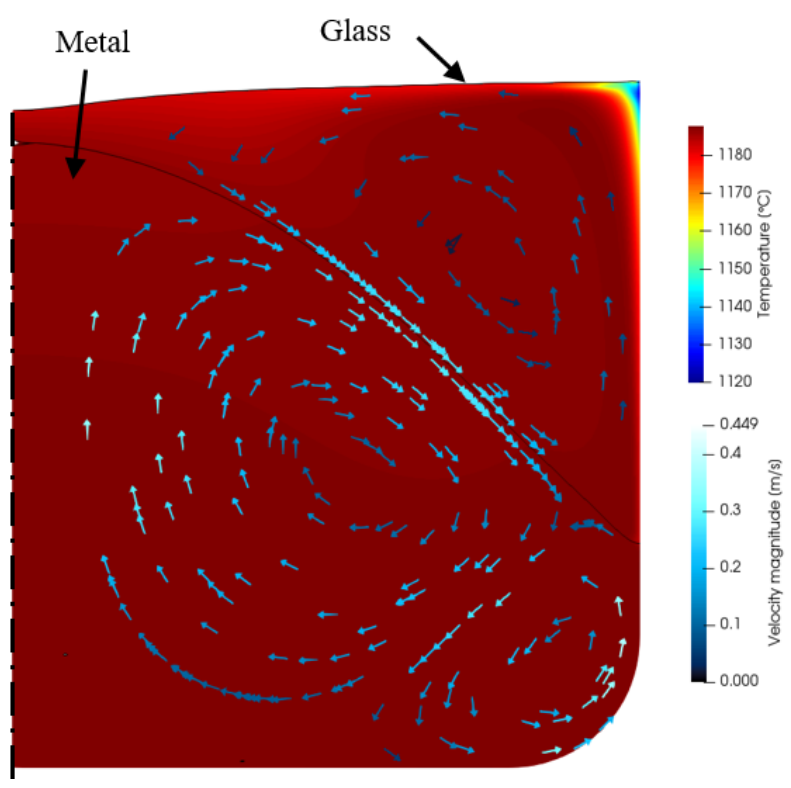

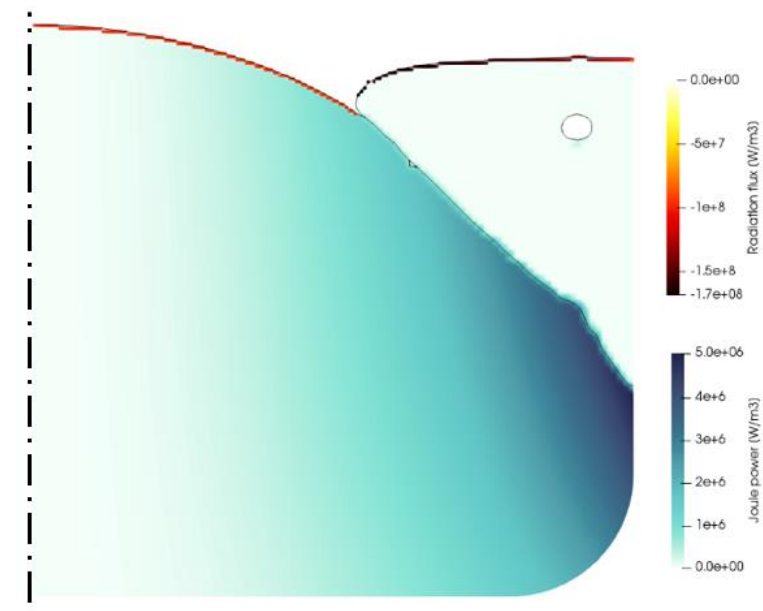

Figure 4. Joule power density and radiative flux in the liquids for $25 \mathrm{~kg}$ of glass

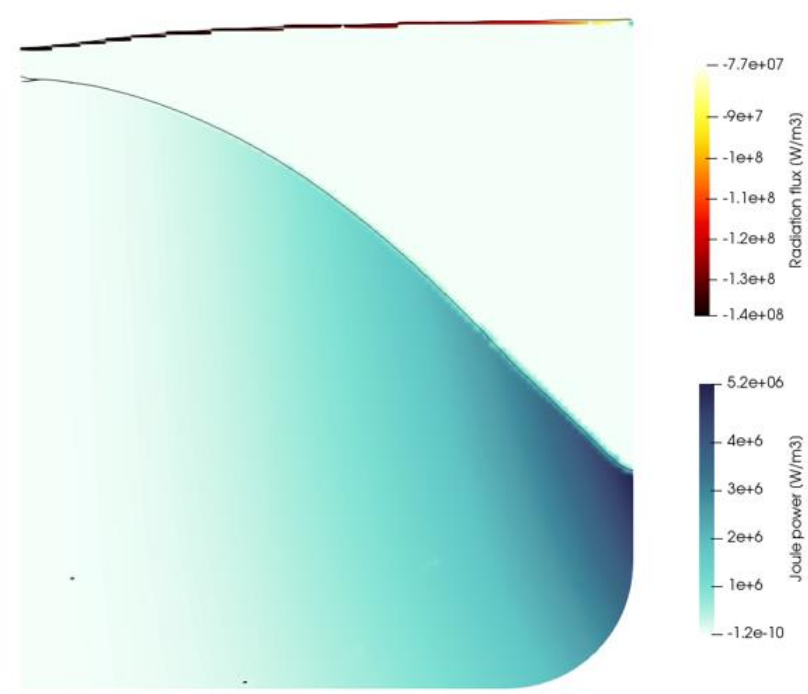


Figure 5. Temperature distribution and velocity vectors in the liquids for $50 \mathrm{~kg}$ of glass
Figure 6. Joule power density and radiative flux in the liquids for $50 \mathrm{~kg}$ of glass

Figures 3-4 and 5-6 represents the results of the numerical model for the same operating conditions but with a doubled quantity of glass. In this case, the glass covers entirely the metallic phase. In this case, we notice that the temperature of the melt is approximately $60{ }^{\circ} \mathrm{C}$ lower, because of the higher radiation fluxes at the free surface. This temperature can't be reached on the prototype because the metal would be under its melting point. This probably comes from an over-estimation of the emissivity of the glass or of the flow of this material. Indeed, the viscosity being strongly dependent on the temperature, this phenomenon should decrease the stirring of this material. This shows the importance to consider this phenomena in the numerical model. Also, the turbulence model chosen for this model may overestimate heat transfers in the materials. LES modelling may be used to prevent this, as pointed out by some authors $[3,4]$.

\section{CONCLUSIONS}

A numerical model able to represent the radiative heat transfers at the free surface of a melt heated and stirred electromagnetically has been implemented in a model using VOF. The cooling fluxes calculated with this numerical model have been validated with the measurements on the prototype of the process, along with the temperature of a point inside the crucible. The results of the temperature distribution in the liquids shows a cool area at the free surface of the glass, probably responsible for the solidification of this material.

Further investigations must be carried out to characterize this phenomenon, using a viscosity of the glass varying with the temperature in the model.

\section{ACKNOLEDGEMENTS}

This work was financially supported by the "Investing for the Future" program of the SGPI (French governmental administration) granted to the ANDRA, by ORANO (former AREVA), and by the CEA.

\section{REFERENCES}

[1] Bourrou, R., Gagnoud, A., Budenkova, O., Charvin, P., and Lafon, C. (2018). "Numerical simulation of surface deformation in a three-fluid process stirred by low frequency magnetic field", in International conference on the electromagnetic processing of materials, Japan, 012030.

[2] Eckert, E.R.G. and Drake R.M. (1960), Heat and Mass Transfer (2 ${ }^{\text {nd }}$ edition), McGRAWHILL.

[3] Baake, E., Nacke, B., Umbrashko, A., and Jakovics, A. (2003), "Turbulent flow dynamics, heat transfer and mass exchange in the melt of induction furnaces", COMPEL, Vol. 22, pp. 39-47.

[4] Umbrashko, A., Baake, E., Nacke, B., and Jakovics, A. (2008), "Numerical studies of the melting process in the induction furnace with cold crucible", COMPEL, Vol. 27, pp. 359368. 